\title{
Checklist de Ephemeroptera do Estado de Mato Grosso do Sul, Brasil
}

\author{
Fábio Henrique da Silva \& Frederico Falcão Salles
}

Departamento de Ciências Agrárias e Biológicas, Laboratório de Sistemática e Ecologia de Insetos, Centro Universitário Norte do Espírito Santo, Universidade Federal do Espírito Santo - UFES, Rodovia BR-101, 29932-540 São Mateus, ES, Brasil. (ambientefhs@gmail.com)

Recebido 24 dezembro 2016

Aceito 6 fevereiro 2017

DOI: $10.1590 / 1678-4766 e 2017116$

ABSTRACT. Checklist of Ephemeroptera of the state of Mato Grosso do Sul, Brazil. The order Ephemeroptera (Insecta) is one of the most important groups of aquatic insect. It is found in freshwater ecosystems lentic and lotic, associated with various substrate types. It's representatives actively participate in nutrient cycling and energy flow and are often used as environmental indicators. The last list of Ephemeroptera to the state of Mato Grosso do Sul recorded only thirteen species representing eight genera and four families. Two researchers are currently working directly with the group in the region.

KEYWORDS. Ephemeroptera, aquatic insects, biodiversity of the State of Mato Grosso do Sul, BIOTA/MS Program.

RESUMO. A ordem Ephemeroptera (Insecta) constitui um dos mais importantes grupos da entomofauna aquática. São encontrados em ambientes aquáticos continentais lênticos e lóticos, associados aos mais diversos tipos de substrato. Seus representantes participam ativamente da ciclagem de nutrientes e do fluxo energético e são muitas vezes utilizados como indicadores ambientais. A última lista de Ephemeroptera para o estado de Mato Grosso do Sul feita registrou apenas treze espécies representantes de oito gêneros e quatro famílias. Atualmente existem dois pesquisadores trabalhando diretamente com o grupo na região.

PALAVRAS-CHAVE. Ephemeroptera, Insetos aquáticos, Biodiversidade do Estado de Mato Grosso do Sul, Programa BIOTA/MS.

A ordem Ephemeroptera é formada por pouco menos de 4.000 espécies de insetos descritas (SAlLes et al., 2004a), sendo que na América do Sul são conhecidas 14 famílias e aproximadamente 450 espécies (Domínguez et al., 2006; Mariano \& Polegato, 2011). Para o Brasil, país da Região Neotropical com o maior número de táxons conhecidos, são registradas dez famílias, 72 gêneros e 318 espécies (SALLES et al., 2014).

Segundo Da-Silva \& Salles (2012) o conhecimento sobre o grupo no Brasil, embora incipiente, vem aumentando consideravelmente nos últimos anos com o aumento de publicações de artigos taxonômicos e de distribuição geográfica, o que contribuiu para que dobrasse o número de espécies reportadas para o Brasil, em duas décadas.

Biologicamente, este grupo se caracteriza pela longa duração do período ninfal, que apresenta estágios imaturos aquáticos e desenvolvimento hemimetábólico (DA-SiLvA et al., 2003), em contraste com a curta vida na fase adulta, exclusivamente destinada à reprodução e dispersão, de onde se origina o nome da ordem (ephemero, de um dia, de vida curta), como aponta BiSPo \& CRISCi-BisPo (2006).

Seus representantes são obrigatoriamente anfibióticos, com imaturos aquáticos e adultos terrestres. Suas ninfas constituem um dos principais grupos dentre os macroinvertebrados bentônicos. Além de serem extremamente abundantes e diversas, ocupam a maior parte dos meso-hábitats disponíveis em todos os tipos de ambientes aquáticos, desde aqueles em áreas de remanso até os de forte correnteza. Como as ninfas são em grande parte herbívoras ou detritívoras, e servem de alimento para uma série de predadores, como outros insetos e peixes, representam um importante elo na cadeia trófica dos ambientes aquáticos (SAlles et al., 2004a).

Entre os diferentes grupos das comunidades aquáticas, as formas imaturas de insetos da ordem Ephemeroptera são importantes componentes na maioria dos ecossistemas de água doce (HUBBARD, 1982; DA-SiLVA et al., 2003), onde participam ativamente da ciclagem de nutrientes e do fluxo energia.

O grupo exibe ainda uma característica peculiar da ordem, a existência de um estágio alado intermediário entre a ninfa e o adulto, denominado subimago ou subadulto, que distintamente dos adultos, frequentemente encontrados revoando sobre ou nas proximidades dos corpos d'água, são menos ativas, e ficam pousadas às margens dos ambientes dos quais emergiram (SALLES et al., 2004a).

Em função das distintas respostas apresentadas por suas espécies à degradação ambiental, e o fato de determinadas famílias terem sua taxonomia baseada no estágio imaturo, ao contrário da maioria das ordens de insetos com representantes aquáticos, os Ephemeroptera são um dos grupos mais utilizados em programas de biomonitoramento de qualidade da água (SALLES et al., 2004a). 
Diversos trabalhos vêm sendo realizados no Brasil com o intuito de levantar dados sobre a composição e a distribuição da fauna de Ephemeroptera em diversos estados brasileiros, sendo poucos os realizados para o Centro-Oeste (e.g. Shimano et al., 2010 ou 2011 para o leste do estado do Mato Grosso). Dessa forma, o presente trabalho tem como objetivo apresentar uma lista das espécies de Ephemeroptera registradas até o momento para o estado de Mato Grosso do Sul, assim como propor medidas para mitigar o parco conhecimento a respeito do grupo na região.

\section{MATERIAL E MÉTODOS}

O checklist foi realizado com base nas publicações de registros de ocorrência de espécies para o estado de Mato Grosso do Sul e na publicação da Lista das espécies de Ephemeroptera registradas para o Brasil (SALLES et al., 2004), a qual frequentemente vem sendo atualizada via internet no site Ephemeroptera do Brasil (www.ephemeroptera.com.br) elaborado por SALLES et al. (2014).

\section{RESULTADOS E DISCUSSÃO}

\section{Lista das espécies de Ephemeroptera do estado de Mato Grosso do Sul, Brasil}

Família Baetidae

Callibaetis jocosus Navás, 1912

Callibaetis pollens Needham \& Murphy, 1924

Callibaetis viviparus Needham \& Murphy, 1924

Camelobaetidius cruzi Boldrini, 2014

Família Leptohyphidae

Amanahyphes saguassu Salles \& Molineri, 2006

Família Leptophlebiidae

Hydromastodon sallesi Polegato \& Batista, 2007

Segesta riograndensis Siegloch \& Polegatto, 2006

Família Polymitarcyidae

Asthenopus angelae De-Souza \& Molineri, 2012

Campsurus albifilum Walker, 1853

Campsurus corumbanus Needham \& Murphy, 1924

Campsurus notatus Needham \& Murphy, 1924

Campsurus striatus Needham \& Murphy, 1924

Tortopus harrisi Traver, 1950

O conhecimento a respeito dos Ephemeroptera pode ser considerado muito abaixo do satisfatório na Região Centro-Oeste. Áreas prioritárias para a compreensão sobre a distribuição dos representantes da ordem no Brasil e, consequentemente, na América do Sul, ainda são as menos conhecidas. A região Centro-Oeste é a segunda maior em extensão do Brasil, a qual ocupa quase $20 \%$ do território nacional, apresenta um número de registros, apesar de crescente, ainda restrito. Não só diversas famílias não possuem espécies nominais relatadas para a região, como o conhecimento nos distintos estados e no Distrito Federal é fragmentado (SALles et al., 2004).

Algumas pesquisas referentes à Baetidae foram desenvolvidas no Mato Grosso (SAlles \& Batista, 2004;
SALLES et al., 2004b), e sobre a ecologia e distribuição do grupo (Shimano et al., 2010, 2011). Já no estado do Mato Grosso do Sul, atualmente, trabalhos sobre a distribuição da ordem no Planalto da Bodoquena estão sendo realizados pelos autores deste manuscrito, mas comparado a outras regiões, ainda pouco tem sido acrescentado ao conhecimento de Ephemeroptera.

Principais grupos de pesquisa. (1) Eduardo Dominguez, Carlos Molineri, Carolina Nieto - CONICET, Facultad de Ciencias Naturales e IML, Fundación Miguel Lillo, Universidad Nacional de Tucumán, Argentina; (2) Elidiomar Ribeiro Da-Silva - Departamento de Zoologia, Universidade Federal do Estado do Rio de Janeiro; (3) Frederico Falcão Salles - Laboratório de Sistemática e Ecologia de Insetos, Departamento de Ciências Agrárias e Biológicas, Centro Universitário Norte do Espírito Santo, Universidade Federal do Espírito Santo - UFES; (4) Janice Peters, Will Flowers e Barton Richard - Entomology, CESTA, Florida Agricultural \& Mechanical University, Tallahassee, FL, USA; (5) Jorge Luis Nessimian - Laboratório de Entomologia, Departamento de Zoologia, Instituto de Biologia, Universidade Federal do Rio de Janeiro; (6) Lucimar Gomes Dias - Departamento de Ciências Biológicas, Facultad de Ciencias Exactas y Naturales Universidad de Caldas, Colombia; (7) Pitágoras Conceição Bispo - Laboratório de Biologia Aquática, Departamento de Ciências Biológicas, Universidade Estadual Paulista, Faculdade de Ci ências e Letras de Assis - FCLA; (8) Rodolfo Mariano L. S. - Departamento de Ciências Agrárias e Ambientais, Universidade Estadual de Santa Cruz - UESC, Ilhéus, BA.

Principais acervos. Coleção Zoológica Norte Capixaba (CZNC), Centro Universitário Norte do Espírito Santo, Universidade Federal do Espírito Santo, São Mateus, ES, Brasil; Florida Agricultural \& Mechanical University (FAMU), Tallahassee, FL, USA; Fundación Miguel Lillo (IML), Tucumán, Argentina; Instituto Nacional de Pesquisa da Amazônia (INPA), Manaus, AM, Brasil; Museu de Zoologia da Universidade de São Paulo (MZSP), São Paulo, SP, Brasil; Universidade Estadual Santa Cruz, Ilhéus, BA, Brasil; Universidade Federal da Bahia, Salvador, BA, Brasil; Coleção Entomológica Prof. José Alfredo Pinheiro Dutra, Universidade Federal do Rio de Janeiro, Rio de Janeiro, RJ, Brasil.

Principais lacunas do conhecimento. O estado de Mato Grosso do Sul se apresenta praticamente inexplorado quanto ao estudo de insetos aquáticos. A maior parte dos trabalhos realizados no estado referente a insetos aquáticos é de cunho ecológico e foram realizados na região do Pantanal, onde podemos citar Righi-Cavallaro et al. (2010a) e SiLVA et al. $(2008 ; 2009 ; 2012)$ e no Planalto da Bodoquena, Righi-Cavallaro et al. (2010b); Dalzochio et al. (2011) e EsCARPINATI et al. (2011). Com enfoque taxonômico, uma nota de distribuição geográfica de uma espécie de Ephemeroptera no estado de MS foi publicada por RighI-CAVALLARO et al. (2008). Trabalhos que resultaram na descrição das espécies atualmente conhecidas para o estado foram feitos por NAVÁs (1912); NEEDHAM \& MuRPHy (1924); 
Traver (1950); Salles \& Molineri (2006); Siegloch et al. (2006); Polegatto \& Batista (2007); De-Souza \& MOLINERI (2012); Molineri \& SALLES (2013) e BoldRINI \& Pes (2014).

Perspectivas de pesquisa para o grupo nos próximos 10 anos. $\mathrm{O}$ incremento aos estudos de fusão de conhecimento taxonômico, de distribuição geográfica e ecológica, a ampliação de conhecimentos filogenéticos, aliados à realização de trabalhos faunísticos em regiões ainda não estudadas, são de grande importância para o avanço do conhecimento do grupo. Ao mesmo tempo, o incentivo na formação de especialistas e a criação de chaves de identificação próprias para o país e/ou ainda regiões devem ser priorizados, com a finalidade de se dar prosseguimento a trabalhos relacionados à fauna de Ephemeroptera.

Agradecimentos. A Fundação de Apoio ao Desenvolvimento do Ensino, Ciências e Tecnologia do Estado de Mato Grosso do Sul (Fundect) e a Superintendência de Ciências e Tecnologia do Estado de Mato Grosso do Sul (Sucitec/MS) pelo convite de participação neste fascículo especial da Iheringia Série Zoologia e o suporte financeiro para sua publicação. A CAPES pela bolsa de doutorado concedida a FHS e ao CNPq por prover uma bolsa de produtividade a FFS.

\section{REFERÊNCIAS BIBLIOGRÁFICAS}

Bispo, P. C. \& Crisci-Bispo, V. L. 2006. Ephemeroptera. In: Costa, C., IDE, S. \& Simonka, C. E. eds. Insetos Imaturos. Metamorfose e Identificação. Ribeirão Preto, Holos, p.55-59.

BoldRINI, R. \& PES, A. M. O. 2014. Five new species of Camelobaetidius Demoulin, 1966 (Ephemeroptera: Baetidae), and redescription of Camelobaetidius mexicanus (Traver \& Edmunds, 1968). Zootaxa 3:545-567.

Dalzochio, M. S.; Costa, J. M. \& UCHOA, M. A. 2011. Diversity of Odonata (Insecta) in lotic systems from Serra da Bodoquena, Mato Grosso do Sul State, Brazil. Revista Brasileira de Entomologia 55(1):88-94.

Da-Silva, E. R.; Salles, F. F.; Nessimian, J. L. \& Coelho, L. B. N. 2003. A identificação das famílias de Ephemeroptera (INSECTA) ocorrentes no Brasil. Boletim do Museu Nacional, Nova Série, Zoologia 508:1-6.

De-Souza, M. R. \& Molineri, C. 2012. The adults and nymphs of Asthenopus angelae new species (Ephemeroptera: Polymitarcyidae) from Argentina, Bolivia, Brazil and Colombia. Zootaxa 3399: 45-52.

Domínguez, E.; Molineri, C.; Pescador, M.; Hubbard, M. D. \& Nieto, C. 2006. Ephemeroptera of South America. Sofia, Pensoft (Aquatic Biodiversity in Latin América, vol. 2).

Escarpinati, S. C.; Roque, F. O.; Medina J. R. P. \& Raizer, J. 2011. Macroinvertebrate community in recreational areas in a karst river (Bonito, Brazil): implications for biomonitoring of tourist activities. Pesquisas em Turismo e Paisagens Cársticas 4:121-130.

HubBard, M. D. 1982. Catálago abreviado de Ephemeroptera da América do Sul. Papéis Avulsos de Zoologia 34(24):257-282.

Mariano, R. \& Polegatto, C. 2011. Checklist of Ephemeroptera from São Paulo State, Brazil. Biota Neotropica 11(1a):291-296.

Molineri, C. \& SAlles, F. F. 2013. Phylogeny and biogeography of the ephemeral Campsurus Eaton (Ephemeroptera, Polymitarcyidae). Systematic Entomology 38(2):265-277.

Navás, L. 1912. Neurópteros nuevos de América. Broteria 10:194-202.

Needham, J. G. \& MurPhy, H. E. 1924. Neotropical mayflies. Bulletin of the Lloyd Library of Botany, Pharmacy and Materia Medica, No. 24, Entomologica Series 4:1-79.
Polegatto, C. M \& Batista, J. D. 2007. Hydromastodon sallesi, new genus and new species of Atalophlebiinae (Insecta: Ephemeroptera: Leptophlebiidae) from West and North of Brazil, and notes on systematics of Hermanella. Zootaxa 1619:53-60.

Righi-Cavallaro, K. O.; Siegloch, A. E.; Calheiros, D. \& Barbosa, D. S. 2008. Segesta riograndensis, Ephemeroptera, Leptophlebiidae, Atalophlebiinae: First record from state of Mato Grosso do Sul, western Brazil. Notes on Geographic Distribution. Check List 4(4):472-474.

Righi-Cavallaro, K. O.; Spies, M. R. \& Siegloch, A. E. 2010a. Ephemeroptera, Plecoptera and Trichoptera assemblages in Miranda River basin, Mato Grosso do Sul State, Brazil. Biota Neotropica 10(2):253-261.

Righi-Cavallaro, K.O.; Roche, K. F.; Froehlich, O. \& Cavallaro, M. R. 2010b. Structure of macroinvertebrate communities in riffles of a Neotropical karst stream in the wet and dry seasons. Acta Limnologica Brasiliensia 22(3):306-316.

Salles, F. F.; Da-Silva, E. R.; Hubbard, M. D. \& Serrão, J. E. 2004a. As espécies de Ephemeroptera (Insecta) registradas para o Brasil. Biota Neotropica 4(2):1-34.

Salles, F. F.; Boldrini, R.; Nascimento, J. C. M.;Raimundi, E. A. \& Shimano, Y. F. 2014. Ephemeroptera do Brasil. Disponível em: $<$ www.ephemeroptera.com.br>. Acessado em: 17.07.2014.

Salles, F. F. \& Batista, J. D. 2004. The presence of Varipes Lugo-Ortiz \& McCafferty (Ephemeroptera: Baetidae) in Brazil, with the description of a new species. Zootaxa 456:1-6.

SAlles, F. F.; Batista, J. D. \& CABEtTe, H. R. S. 2004b. Baetidae (Insecta: Ephemeroptera) de Nova Xavantina, Mato Grosso, Brasil: novos registros e descrição de uma nova espécie de Cloeodes Traver. Biota Neotropica 4(2):1-8.

SAlles, F. F. \& Molineri, C. 2006. Amanahyphes saguassu, a new genus and species of Leptohyphidae (Ephemeroptera: Ephemerelloidea) from northern Brazil. Aquatic Insects 28:1-12.

Shimano, Y.; Cabette, H. S. R.; SAlles, F. F. \& Juen, L. 2010. Composição e distribuição da fauna de Ephemeroptera (Insecta) em área de transição Cerrado-Amazônia, Brasil. Iheringia, Série Zoologia 100(4):301-308.

Shimano, Y.; Salles, F. F. \& Cabette, H. S. R. 2011. Ephemeroptera (Insecta) ocorrentes no Leste do Estado do Mato Grosso, Brasil. Biota Neotropica 11(4):239-253.

Siegloch, A. E.; Polegatto, C. M. \& Froehlich, C. G. 2006. Segesta riograndensis, new genus and species of an Atalophlebiinae (Ephemeroptera: Leptophlebiidae) mayfly from southern Brazil. Zootaxa 1299:35-43.

SiLVA, E. R. \& SALLES, F. F. 2012. Ephemeroptera Hyatt \& Arms, 1891. In: Rafael, J. A.; Melo, G. A. R.; Carvalho, C. J. B.; Casari, S. A. \& Constantino, R. eds. Insetos do Brasil Diversidade e Taxonomia. Ribeirão Preto, Holos, p.231-243.

Silva, F. H.; FAVERO, S. \& SABINO, J. 2012. Functional groups of entomofauna associated to aquatic macrophytes in Correntoso river, Rio Negro subregion, Pantanal, Mato Grosso do Sul State, Brazil. Acta Scientiarum, Biological Sciences 34(1):59-68.

Silva, F. H.; FaVero, S.; Sabino, J. \& GarnÉs, S. J. A. 2008. Estrutura da comunidade de insetos associados a macrófitas aquáticas, em um trecho do rio Correntoso, Pantanal do Negro, Mato Grosso do Sul, Brasil. In: Oliveira, A. K. M; Garnés, S. J. A. \& Figueiredo, R. S. eds. Meio ambiente e produção interdisciplinar: sociedade, natureza e desenvolvimento. Campo Grande, Uniderp, p. 99-117.

Silva, F. H.; FAVERo, S.; SABINO, J. \& GARNÉs, S. J. A. 2009. Distribuição da entomofauna associada às macrófitas aquáticas na vazante do rio Correntoso, Pantanal do Negro, Estado do Mato Grosso do Sul, Brasil. Acta Scientiarum, Biological Sciences 31(2):127-134.

Traver, J. R. 1950. Notes on Neotropical mayflies. Part. IV. Family Ephemeridae (continued). Revista de Entomologia 21:593-614. 